\title{
Re-designing Urban Stream Landscape by Investigating the Citizens' Preference Matrix
}

\author{
Ali Asadpour \\ Faculty of Architecture \& Environmental Design, Shiraz University of Arts, Iran
}

Copyright $(\mathrm{C} 2017$ by authors, all rights reserved. Authors agree that this article remains permanently open access under the terms of the Creative Commons Attribution License 4.0 International License

\begin{abstract}
In addition to the ecological concerns, public preferences and social perceptions are important subjects in urban stream redevelopment. There are few studies addressing public concerns in Middle East nations, such as Iran. This study took The Khoshk River (in local language means Dry River) in Shiraz metropolitan area, Iran, as an object to do some comprehensive evaluation of its natural and built environment by questioner survey. Random sampling method have been used and totally 474 citizens participated in the study. They had been asked to choose the river scape preference according to Kaplan's' environmental matrix (coherence, legibility, complexity \& mystery) in Likert scale. The variable 'naturalness' added to the mentioned matrix as a proposal. Findings analyzed by SPSS (Version 19). Results show that there is a significant correlation between naturalness and 'coherence', 'complexity', 'legibility' and 'mystery' preference named in order of importance and relationships. It means that, naturalness as a preference variable has a grater correlation with 'immediate' variables (named coherence and complexity) than inferred ones (named legibility and mystery). The paper, suggests that naturalness could be added to preference matrix of urban streams. Furthermore, the results confirm the gaps between public perceptions and purpose of city governments in naturalizing the river scape. Strategies are also being proposed to redesign the landscape of the river.
\end{abstract}

Keywords Preference Matrix, Urban Stream, Landscape, Naturalness, Khoshk River

\section{Introduction}

Increasing urbanization across landscapes of the world has led to increased researches on ecology in urban settings in the last 1 to 2 decades [1]. Besides the ecological concern, public preferences and social perceptions are important subjects in urban landscape redevelopment. Rivers have had a crucial part in the emergence of human society, being strongly present in almost every single stage of the human journey, and heavily implicated in the process of human settlement [2]. In this study, an urban stream as an urban landscape, defined as a formerly natural waterway that flows through a heavily populated area, which is often significantly polluted, due to urban runoff and combined sewer outflows [3]. Rehabilitation of urban streams is a well-established worldwide trend and It is regarded as an effective way to promote the regeneration and development of cities [3] and numerous studies have addressed this issue basically with ecological concerns. However, there are few studies focusing on public preferences in Middle East nations with hot or semi hot arid conditions. For instance, Che et al. (2012), focus on the concept of accessibility in developing a method to assess the riverfront after rehabilitation of an urban stream in Shanghai, China, using a comprehensive index based on the consideration that the accessibility of urban riverfronts is significant in terms of realizing ecological and social benefits of urban riverfronts. Le Lay et al. (2013) studied the ecologically-diverse incising of braided Magra River in Italy. They identifies a distance between scientific and popular attitudes and discusses implications for public participation, support for braided river restoration policy, and environmental education [4]. $W u$ et al. (2011), assess the perception and use of East Ho Chung River greenway in Guangzhou, China to provides a comprehensive evaluations towards a goal of establishing a multi-functional river corridor greenway that meets people's needs and preferences [5]. Nagpal \& Sinha (2009), outlines an urban conservation model and suggests design interventions that would revitalize the Gomti riverfront in Lucknow, India and contribute towards preserving both tangible and intangible heritage of the city [6]. Less than two decades ago, Tapsell (1997), discussed the rivers and river restoration according to child's eye views [7].

In 2016 Jonathan Prior analyses the restoration of an urbanized section of the River Skerne where it flows through a suburb of Darlington, England; a project which was one of the first comprehensive urban river restorations undertaken 
in the UK. It is shown how aesthetic values were central to the identification of the River Skerne as a site for restoration, the production of restoration objectives, and a design vision of urban river renewal via restoration [8]. Pinto \& Maheshwari (2016) examine aspects of community values and perspectives on goods and services received from the river along with issues related to sustainable management of the river. That study reveals that the contemporary values placed on rivers by communities are mostly associated with recreational and leisure activities [9]. Podolak \& Kondolf (2016) tries to find the line of beauty in river designs in Hogarth's Aesthetic Theory and Capability Brown's eighteenth-century river design and twentieth-century river restoration design. They found that Brown's features matched Hogarth's line in sinuosity but were asymmetrical, while the recent stream restoration project designs had higher sinuosities and were nearly perfectly symmetrical [10].

The mentioned studies emphasize the importance of human perceptions in any urban stream interventions. Human perception appears to be critical for understanding the interactions between humans and landscape. Through their actions, people affect the landscape, and in turn, the landscape affects people by means of its appearance. People received landscapes and reflect upon them. Understanding the interactions between humans and landscape is thus essential to sustainable landscape planning and management in greenway planning [5]. In the other words, A fundamental set of constructs in landscape design and environmental psychology deal with how humans perceive nature, how they affect nature, and in turn, how they are affected by nature [11]. On the other hands, differences in preference between experts and other groups can be particularly problematic in situations where experts are making decisions about landscape aesthetics on behalf of the public or other interest groups. Researches show that not only do certain groups tend to see things differently, but they often hold misconceptions about the perspectives of other groups [12].

This paper took Khoshk River in Shiraz metropolitan area, Iran, as an object to do some comprehensive evaluation of its natural and built environment by the method of social survey. This study aims to improve the quality of urban streams environment, and to make the design and interventions more citizens' oriented. In the following pages, the variables of the study would be described more fully, detailing the methods from which the results would be draw.

\section{Method \& Study Area}

Shiraz city with a population of more than 1.8 million people is an important historical, cultural, social, and economic center in southern Iran. The earliest reference to the city is on Elamite clay tablets dated to $2000 \mathrm{BC}$. The city was the Iranian (Persian) capital during the Zand dynasty from $1750 \mathrm{AD}$ until $1781 \mathrm{AD}$. One of the largest cities in Iran and the capital of the Fars province, Shiraz cover a land area of about $340 \mathrm{~km}^{2}$. With an average elevation of $1500 \mathrm{~m}$ above sea level, it is located $\left(29 \_530 \mathrm{~N}, 52 \_580 \mathrm{E}\right)$ in a NWSE elongated valley bounded by the Zagros Mountains [13]. The Khoshk River (in local language Dry River) flows through the northern part of the city seasonally, ending in the Maharlou Lake to the East. Shiraz has a moderate climate with four regular seasons. An analysis of shiraz large-scale historical landscape based on images drawn by foreign travelers in about 200 years period, shows that the Khoshk river is one of the tree key natural element of the city historical landscape in drawings [11]. The river basin divided in three zones (Figure 1); the first zone (A) called the upper zone which located at the northern part of the city surrounded by private gardens and in some part mountain scape. Yet, minimum residential tissue exists. The middle zone (B), located near historical urban context, no significant vegetation exist in this part. The lower zone of the river (C) go along the southern part of the city and across along the agricultural lands to lead the Maharlou lake. The stream as its name indicates contains water only in rainy seasons. So it has a paradoxical name and condition. The river limits defined by the stone wall and many traffic bridges connected the two city parts. About a decade ago, Shiraz Municipality started a program called 'pine vegetation' along the riverside in order to increase the natural richness of the river and also hide the dry bed of the stream especially in summer seasons (Figure 2). 

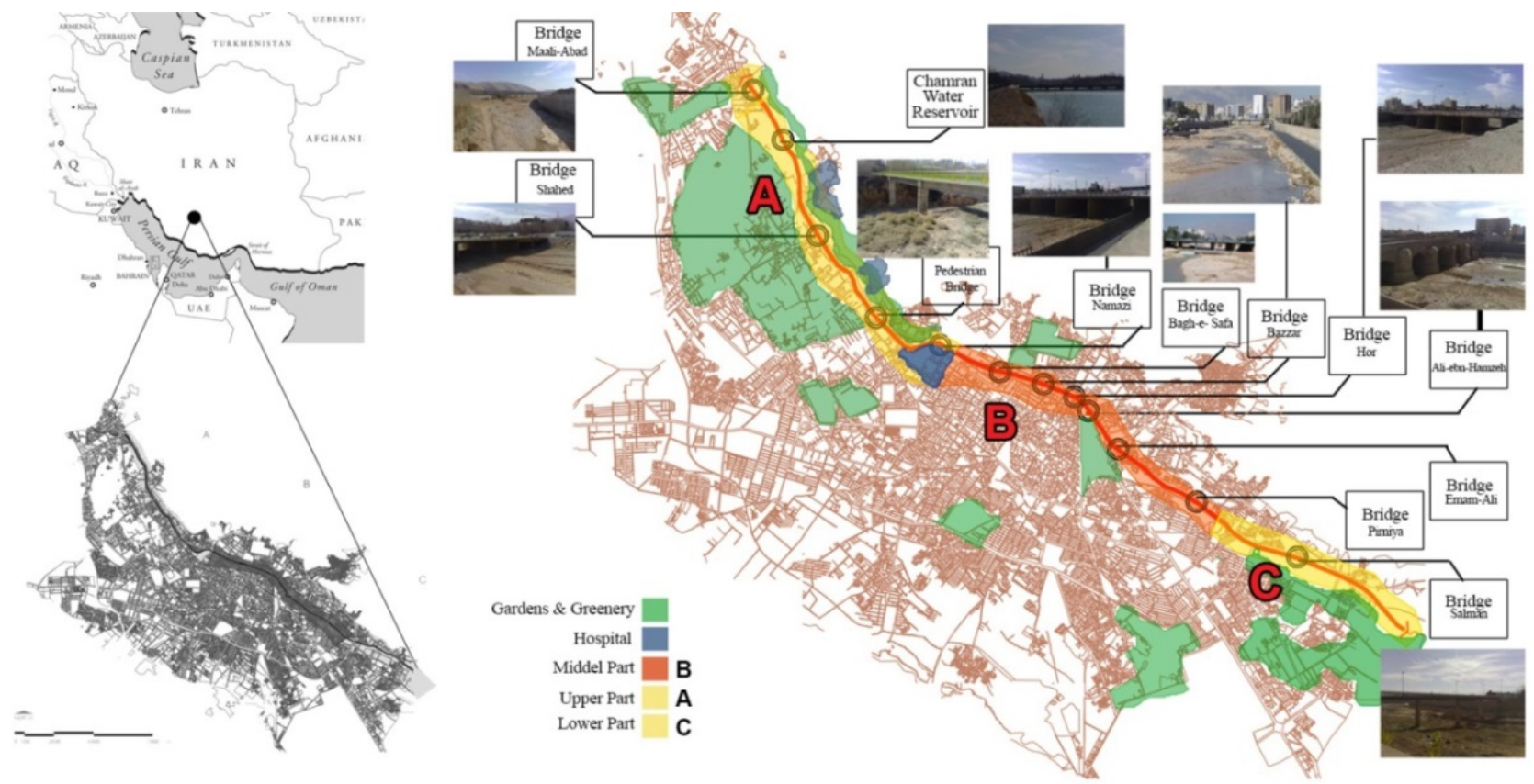

Figure 1. The Khoshk River zoning in Shiraz city, Fars Province, IRAN (with edition)[14]
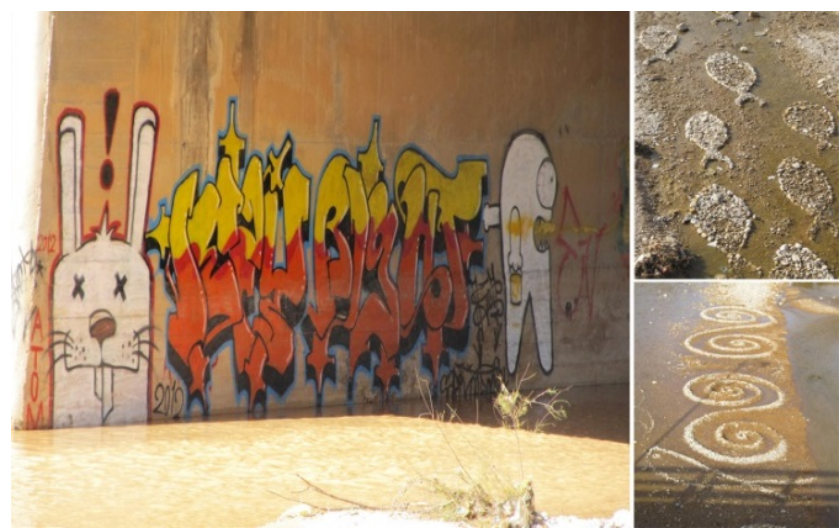

Figure 2. The Khoshk River land arts \& gratifies

This study took place in public spaces such as parks and public gardens (Melli, Azadi, Chamran and Eram), bazaars and commercials complexes (Enghelab and Vakil) and main streets along the riverside, from 15th March 2015 to 25th Juan 2016. We used questionnaire based survey to achieve citizens' preference about the river landscape with random sampling method. As, not many interventions accrued in the upper zone of the stream (part A), we took 17 panorama pictures along the riverside of this area as our field study part. These photos consider all episode of the river landscape which could be seen by foot from the walkway line along the river (Figure 3). The photos printed and attached to the questionnaires. The questionnaire consists of 2 main parts. Part (A), address the personal profiles such as gender, age, education and income. The second part (B), assess the river environmental preferences of the users in Likert scale by five questions about naturalness, legibility, coherence, mystery and complexity which have been mentioned in theoretical framework comes bellow. Data analyzed by SPSS 19 .
Totally, 520 questionnaires were distributed and got returned $474(91.2 \%)$. Of the 474 individuals who participated, 241 $(49.7 \%)$ were men and $233(48 \%)$ were women. Age, education and income of participations, is shown in the following Table 1.

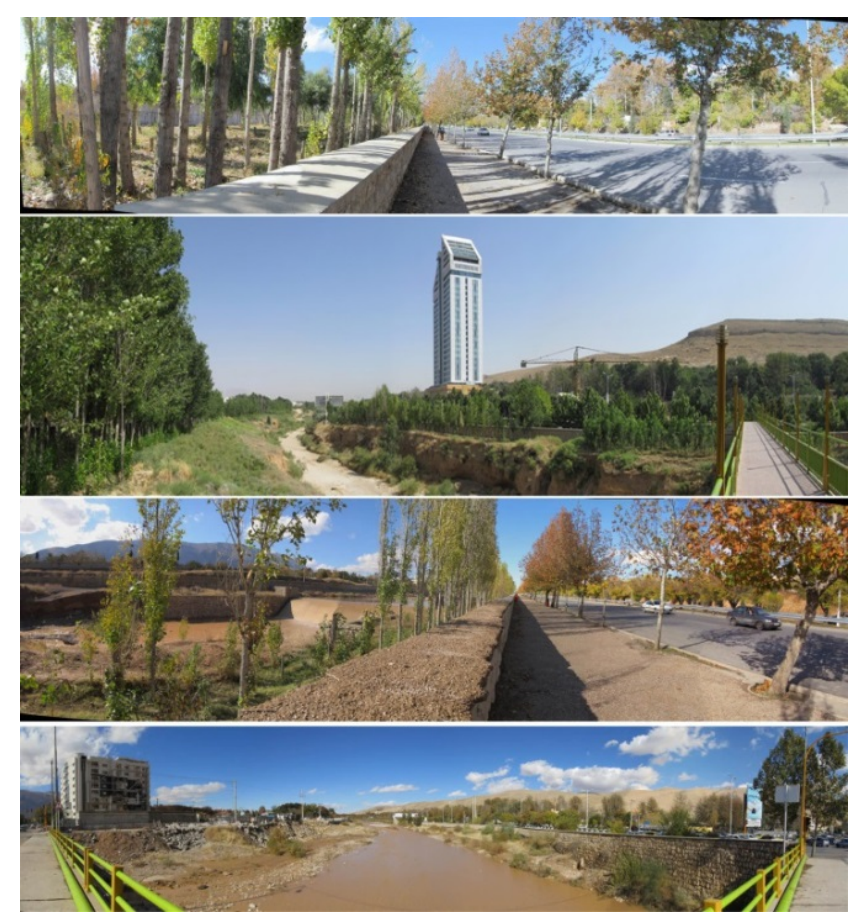

Figure 3. The Khoshk River landscape panoramas

\section{Theoretical Framework}

A model of environmental preference was developed by 
Steven and Rachel Kaplan in 1987 and is known as the Kaplan and Kaplan preference model [15]. But, one of the first people to do this was Berlyne (1960), who conducted a number of studies trying to identify what made a scene aesthetically pleasing. The first series of studies identified four basic properties (complexity, novelty, congruity \& surprisingness) of environments that allow us to evaluate them in comparison to other scenes that we have experienced (called collative properties) [16]. The problem with his method is that two different scenes may score the same on Berlyne's collative properties, but one might be a cityscape whilst the other may be a picture of the countryside. The individual preference for these may relate to past experiences, for example people born in the city may prefer the cityscape. An alternative model of environmental preference, Kaplan's' environmental preference matrix (Table 2), is similar to Berlyne's collative properties, the difference is that the Kaplan's believe a scene has to encourage a certain degree of cognitive processing in order to make it more appealing [16]. Their model consists of four variables named legibility, coherence, mystery and complexity the first two emerged from environment understanding while the last two components needs exploration in landscape. The Understanding and Exploration vector of the preference matrix can be regarded as experiential conceptualizations of objective attributes such as: "uniformity and variety" as well as "order and complexity" [17].

Table 1. Participations attribute

\begin{tabular}{|c|c|c|}
\hline Item & Study Sample & Number \\
\hline \multirow{2}{*}{ Gender } & Male: $49.7 \%$ & 241 \\
\hline & Female: $48 \%$ & 233 \\
\hline \multirow{4}{*}{ Education } & High school: $34.2 \%$ & 162 \\
\hline & Bachelor degree: $51.5 \%$ & 244 \\
\hline & Master degree: $10.5 \%$ & 50 \\
\hline & $\mathrm{PhD}: 3.8 \%$ & 18 \\
\hline \multirow{7}{*}{ Occupation } & Governmental employee: $26 \%$ & 123 \\
\hline & Worker: $0.6 \%$ & 3 \\
\hline & Self-employee: $20.1 \%$ & 95 \\
\hline & Student: $28.8 \%$ & 136 \\
\hline & Home worker: $9.5 \%$ & 45 \\
\hline & No job: $5.5 \%$ & 26 \\
\hline & Retired: $9.5 \%$ & 45 \\
\hline \multirow{6}{*}{ Age } & From $18-27$ years old: $41.1 \%$ & 198 \\
\hline & From $28-37$ years old: $22.6 \%$ & 110 \\
\hline & From $38-47$ years old: $13.3 \%$ & 66 \\
\hline & From $48-57$ years old: $12.7 \%$ & 64 \\
\hline & From $58-67$ years old: $6.3 \%$ & 33 \\
\hline & 68 and more years old: $4 \%$ & 3 \\
\hline \multirow{3}{*}{ Income } & Less than $600 \$$ monthly: $8.4 \%$ & 40 \\
\hline & Between $600 \$$ to $1200 \$$ monthly: $84.8 \%$ & 402 \\
\hline & More than $1200 \$$ monthly: $6.8 \%$ & 32 \\
\hline
\end{tabular}

Table 2. The Kaplan's' preference matrix

\begin{tabular}{|c|c|c|}
\hline & Understanding & Exploration \\
\hline Immediate & Coherence & Complexity \\
\hline Inferred & Legibility & Mystery \\
\hline
\end{tabular}

\section{Study Variables}

Coherence in Kaplan's' model accrued when the visual elements of the scene fit together well. Kaplan \& Kaplan (1989) suggest that a coherent landscape setting contributes to one's ability to make sense of the environment through providing a sense of order and directing the attention of the observer. It also defines as a reflection of the unity of a scene, where coherence may be enhanced through repeating patterns of color and texture. Coherence is also a reflection of the correspondence between land use and natural conditions in an area $[15,17,18]$. Complexity (the scene contains diverse elements and features) has been identified as a key concept of visual quality. It defined as the diversity and richness of landscape elements and features, their interspersion as well as the grain size of the landscape [18].

Legibility would be easiness of finding the way around the environment depicted. The term used by Lynch (1960) to define the well-arranged component of the city elements in an integrated image in the citizens' mind [19]. While, legibility needs some kinds of systematic order, Mystery would be an interesting scene to explore further. How much does a scene promise more if you could walk deeper into it? [20]. Mystery is defined as the promise of new information if one could travel deeper into the environment. Kaplan and Kaplan (1983) discussed that landscapes with mystery tend to invoke inferential mental activity such as an exploratory response in the observer, which enhances preference for the landscape [21]. In this study, we added naturalness to Kaplan's' model. This variable is essential in our case study, because of some interventions made along the riverfront in the past three decades. Naturalness is closeness to a preconceived natural state. The concept of naturalness is linked to evolutionary theories of preferences. It is generally used to describe how close a landscape is to a perceived natural state. Perceived naturalness can thus be different from ecological naturalness [18]. Thus, in this survey, we just emphasize on the visual aspects of naturalness.

\section{Results}

Statically, from 474 returned questionnaires, most of the respondents prefer the river landscape 'coherence' as very low and low (58.4\%), 30.5\% think it's 'coherence' is average and only $11 \%$ prefer it as high and very high. The same results achieved for the variable 'legibility', 46.2\% rank the landscape as low and very low level. While, $22.5 \%$ of respondents ranked this item as high and very high condition. Rests of them $(31.2 \%)$ think that the river scape legibility is in average condition (Figure 4). Citizens' preferences about 
the variable 'complexity' shows that only $12.1 \%$ fined the scape in high and very high level of complexity, $31.6 \%$ in average level and $56.3 \%$ in low and very low condition. Statically, $21.7 \%$ of respondent think the river landscape has high and very high 'mystery' scenes or parts. Oppositely, $51.3 \%$ find the scape with low and very low degree of mystery. Others, $27 \%$ find it in average condition (Figure 5). Analyzing the variable 'naturalness' shows same results; most of the respondents (26.4\%) find the river scape in low and very low organic condition. On the other hand, $14.1 \%$ thinks the scape is in high and very high and $30.1 \%$ in average condition. Descriptively, all of variables have been ranked at low and very low degree of preferences.

Data analyses were carried out using Pearson correlation test in SPSS 19, which enable the comparison of the variables. In line with this, a chi-square $\left(\chi^{2}\right)$ test was carried out to obtain the significance of the research data (Tables 3 to 6). The variables of 'naturalness' and 'coherence' preferences are related variables. According to table 7, increasing in the natural preferences of the river correlated to its landscape's 'coherence' (Pearson correlation: 0.448, significant at the 0.01 level). When the results from chi square $\left(\chi^{2}\right)$ correlation test were considered, statistically significant differences were found between the naturalness preference of the river and other 3 preference variables; A relationship exists between the 'naturalness' preference level and river 'legibility'. People with very low levels of naturalness preference has been assessed the legibility of the river very low too (Table 4). As a result, for legibility, there were statistically significant differences between level of naturalness and level of legibility $(p=0.000<0.001)$. This variable has a positive correlation with level of naturalness preference which has been measured 0.389 significant at the 0.01 level. Another significant relationship can be found between 'naturalness preference' and 'complexity \& mystery level' of the users' preference. People who have had preferred the river landscape complexity \& mysteriousness in low \& very low condition, fined its naturalness condition out at the same level, too. Pearson correlation between these two variables is significant at the 0.01 level. The value for organic- complexity is 0.395 and for organic- mystery is 0.290 (Tables 5 \& 6). Research also showed a significant differences between "naturalness" preference and respondents 'sex' $(p=0.036<0.05$, Pearson Chi-Square Value: $10.29, \mathrm{df}=4)$. But, the correlation value $(0.011)$ is not considerable. No difference was found for income groups of users $(p=0.163>0.05)$ among different levels of river naturalness preference.
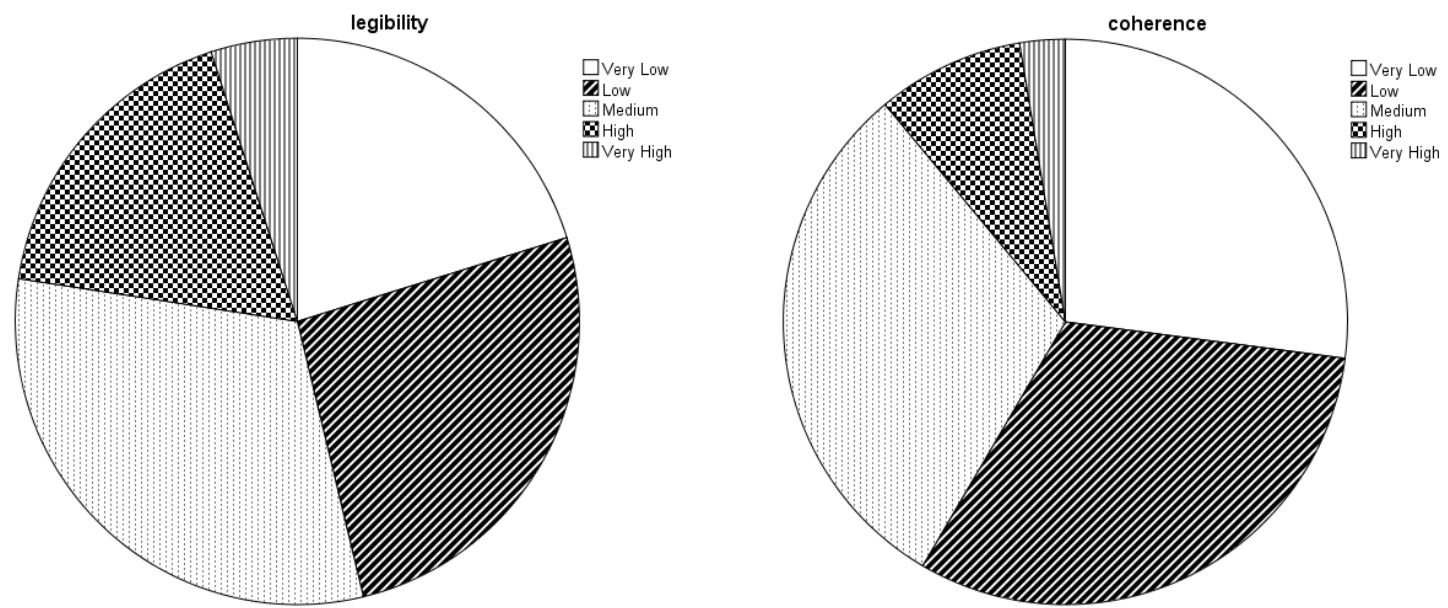

Figure 4. Respondents' preferences about legibility and coherence of the river landscape
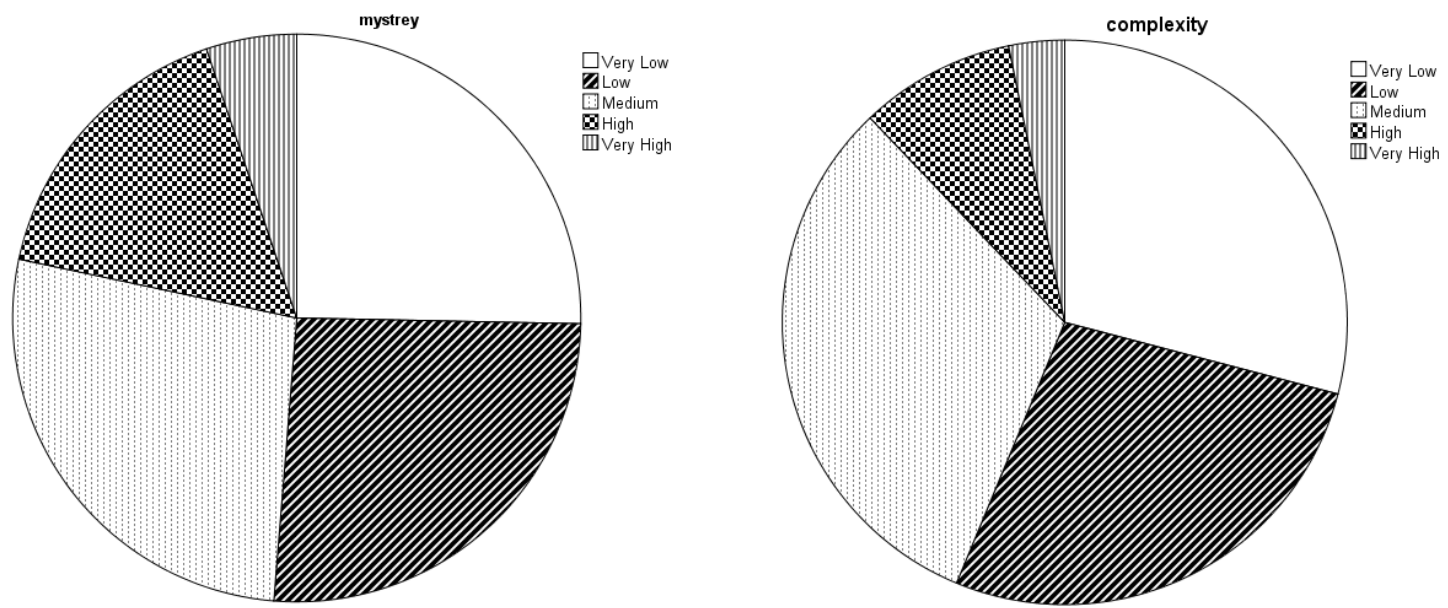

Figure 5. Respondents' preferences about mystery and complexity of the river landscape 


\section{Discussions}

In this study, individual preferences matrix of users based on Kaplan's' preference model (legibility, coherence, mystery and complexity) and differences among these features with the 'naturalness' preference of the Shiraz Khoshk river attempted to be determined. The survey includes series of questions dealing with users' preference and personal features (gender, age, main occupations, and income \& educational level). Although it was expected that the increasing in level of complexity tend to higher level of mysteriousness (Pearson correlation: 0.402), but the results shown in Table 7, verified the relationships between 'naturalness' preference of the citizens and 'coherence', 'complexity', 'legibility' and 'mystery' preference named in order of importance and relationships. It means that, naturalness as a preference variable has a grater correlation with 'immediate' variables (named coherence and complexity) than inferred ones (named legibility and mystery). This could be justified due to the lack of public access to the river bed due to stone walls.

On the other hand, the main objective of the city municipality at first was to redefine the river side scape more naturally in order to hide the unseemly spectacle toward the stream by planting pine trees alongside the river stone retaining walls. Ecologically, these kinds of vegetation seem to be not exactly suited to the river condition [22] but our results emphasize effects of naturalness on increasing other environmental preference factors. In the other words, the objectives of experts and what the citizens preferred at least in this case study seems to be deferred. It means that, the purpose of urban experts was visual pollution removal of the stream by planting pine trees while the citizens used them to increase the environmental preferences such as coherence and complexity. Although, our results did not confirm that planting the trees is the main factor of naturalness preference but implicitly it express that any attempt to make the rivers cape seems more natural could has positive effect on other environmental preferences.

Table 3. Naturalness and coherence variables

\begin{tabular}{|c|c|c|c|c|c|c|c|}
\hline & \multicolumn{5}{|c|}{ Coherence (n.) } & \multirow{2}{*}{ Total } \\
\hline & & Very Low & Low & Medium & High & Very High & \\
\hline \multirow{5}{*}{ Naturalness } & Very Low & 77 & 34 & 17 & 3 & 4 & 135 \\
\hline & Low & 22 & 57 & 36 & 4 & 1 & 120 \\
\hline & Medium & 14 & 48 & 57 & 19 & 1 & 139 \\
\hline & High & 6 & 4 & 25 & 9 & 1 & 45 \\
\hline & Very High & 3 & 2 & 4 & 4 & 5 & 18 \\
\hline \multicolumn{2}{|c|}{ Total } & 122 & 145 & 139 & 39 & 12 & 457 \\
\hline
\end{tabular}

$\mathrm{X}^{2}$-Test $($ organic $\times$ level of coherence $)=190.26, \mathrm{df}=16, p=0.000<0.001$

Table 4. Naturalness and legibility variables

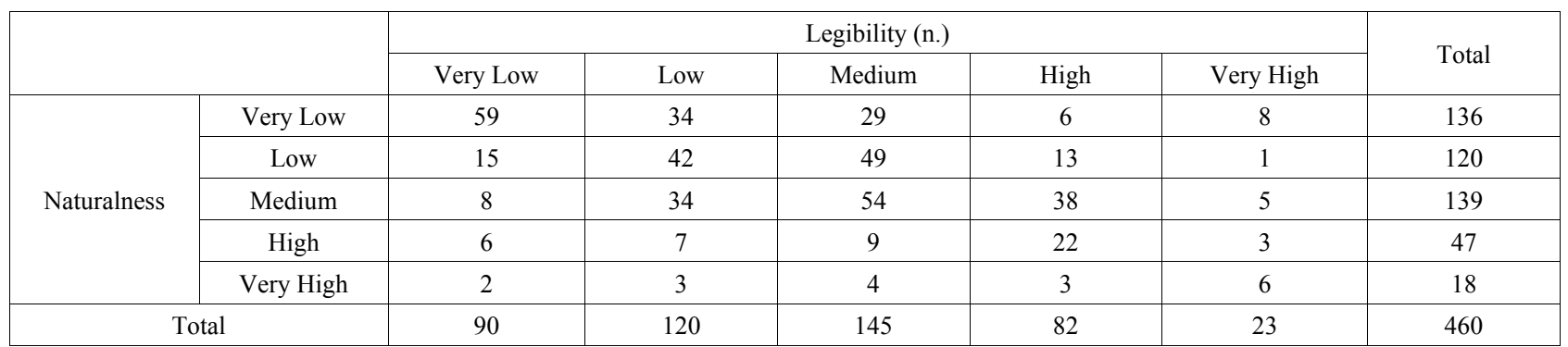

$\mathrm{X}^{2}$-Test $($ organic $\times$ level of legibility $)=156.70, \mathrm{df}=16, p=0.000<0.001$

Table 5. Naturalness and complexity variables

\begin{tabular}{|c|c|c|c|c|c|c|c|}
\hline & & \multicolumn{5}{|c|}{ Complexity (n.) } & \multirow{2}{*}{ Total } \\
\hline & & Very Low & Low & Medium & High & Very High & \\
\hline \multirow{5}{*}{ Naturalness } & Very Low & 71 & 31 & 29 & 4 & 2 & 137 \\
\hline & Low & 39 & 39 & 36 & 8 & 1 & 123 \\
\hline & Medium & 17 & 44 & 57 & 16 & 6 & 140 \\
\hline & High & 6 & 9 & 18 & 11 & 3 & 47 \\
\hline & Very High & 2 & 2 & 9 & 3 & 3 & 19 \\
\hline \multicolumn{2}{|c|}{ Total } & 135 & 125 & 149 & 42 & 15 & 466 \\
\hline
\end{tabular}

$\mathrm{X}^{2}$-Test $($ organic $\times$ level of complexity $)=95.82, \mathrm{df}=16, p=0.000<0.001$ 
Table 6. Naturalness and mystery variables

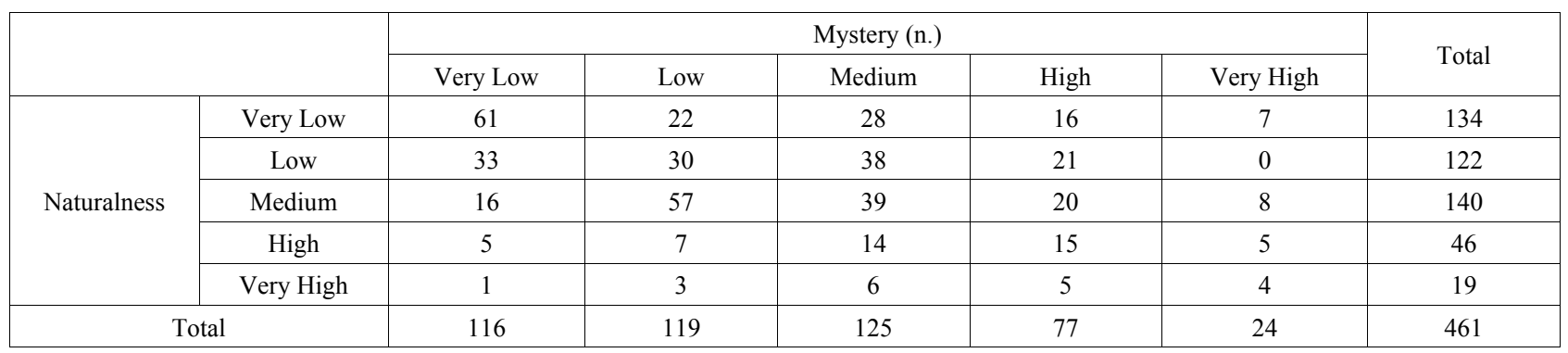

$\mathrm{X}^{2}$-Test (naturalness $\times$ level of mystery) $=90.66, \mathrm{df}=16, p=0.000<0.001$

Table 7. Pearson correlation between variables

\begin{tabular}{|c|c|c|c|c|c|c|}
\hline & & naturalness & coherence & legibility & complexity & mystery \\
\hline \multirow{3}{*}{ naturalness } & Pearson Correlation & 1 & $.448^{* *}$ & $.389^{* *}$ & $.395^{* *}$ & $.290^{* *}$ \\
\hline & Sig. (2-tailed) & & .000 & .000 & .000 & .000 \\
\hline & $\mathrm{N}$ & 469 & 457 & 460 & 466 & 461 \\
\hline \multirow{3}{*}{ coherence } & Pearson Correlation & $.448^{* *}$ & 1 & $.390^{* *}$ & $.268^{* *}$ & $.141^{* *}$ \\
\hline & Sig. (2-tailed) & .000 & & .000 & .000 & .003 \\
\hline & $\mathrm{N}$ & 457 & 462 & 454 & 460 & 455 \\
\hline \multirow{3}{*}{ legibility } & Pearson Correlation & $.389^{* *}$ & $.390^{* *}$ & 1 & $.391^{* *}$ & $.234^{* *}$ \\
\hline & Sig. (2-tailed) & .000 & .000 & & .000 & .000 \\
\hline & $\mathrm{N}$ & 460 & 454 & 465 & 462 & 457 \\
\hline \multirow{3}{*}{ complexity } & Pearson Correlation & $.395^{* *}$ & $.268^{* *}$ & $.391^{* *}$ & 1 & $.402^{* *}$ \\
\hline & Sig. (2-tailed) & .000 & .000 & .000 & & .000 \\
\hline & $\mathrm{N}$ & 466 & 460 & 462 & 471 & 463 \\
\hline \multirow{3}{*}{ mystery } & Pearson Correlation & $.290^{* *}$ & $.141^{* *}$ & $.234^{* *}$ & $.402^{* *}$ & 1 \\
\hline & Sig. (2-tailed) & .000 & .003 & .000 & .000 & \\
\hline & $\mathrm{N}$ & 461 & 455 & 457 & 463 & 466 \\
\hline
\end{tabular}

**. Correlation is significant at the 0.01 level (2-tailed).

*. Correlation is significant at the 0.05 level (2-tailed).

Table 8 shows the images and sections of the river that have the most environmental preferences. As we can see, green spaces play an important role in the perception of the Mystery component. The organic characteristics of the river in connection with water and tree coverings have also been addressed in Figure 6 of Table 8 by the citizens in understanding the Complexity component. Figure 6 in Table 8 has had the most Legibility. There are very few vegetable coatings in this image. The order in the planting of trees on the river's margin and the flow of water has been taken into consideration by citizens in Figure 16 of this table. In their view, this image has had the highest degree of Coherence. It should be noted that the river has very little water, and the presence of water in the river in the seasons will attract people's attention. People care about the plants and birds in the river bed and stand to watch it along the river. However, the stone edges of the river do not always allow this.

\section{Conclusions}

The study adds evidence to the growing body of knowledge in urban stream intervention regarding the associations between naturalness and other four Kaplan's' preference matrix variables. This survey shows the importance of citizens' perceptions and preferences in any urban landscape interventions. And also, emphasized the positive roll of any attempts to increase natural order and appearance of urban streams on citizens' environmental preferences. Finally, as the sum, the listed below could be summarized as main conclusions to increase public preferences in any kinds of interventions in landscape of urban streams: 
Table 8. Evaluation of Kaplan's preference factors contributing to identify character of the river landscape

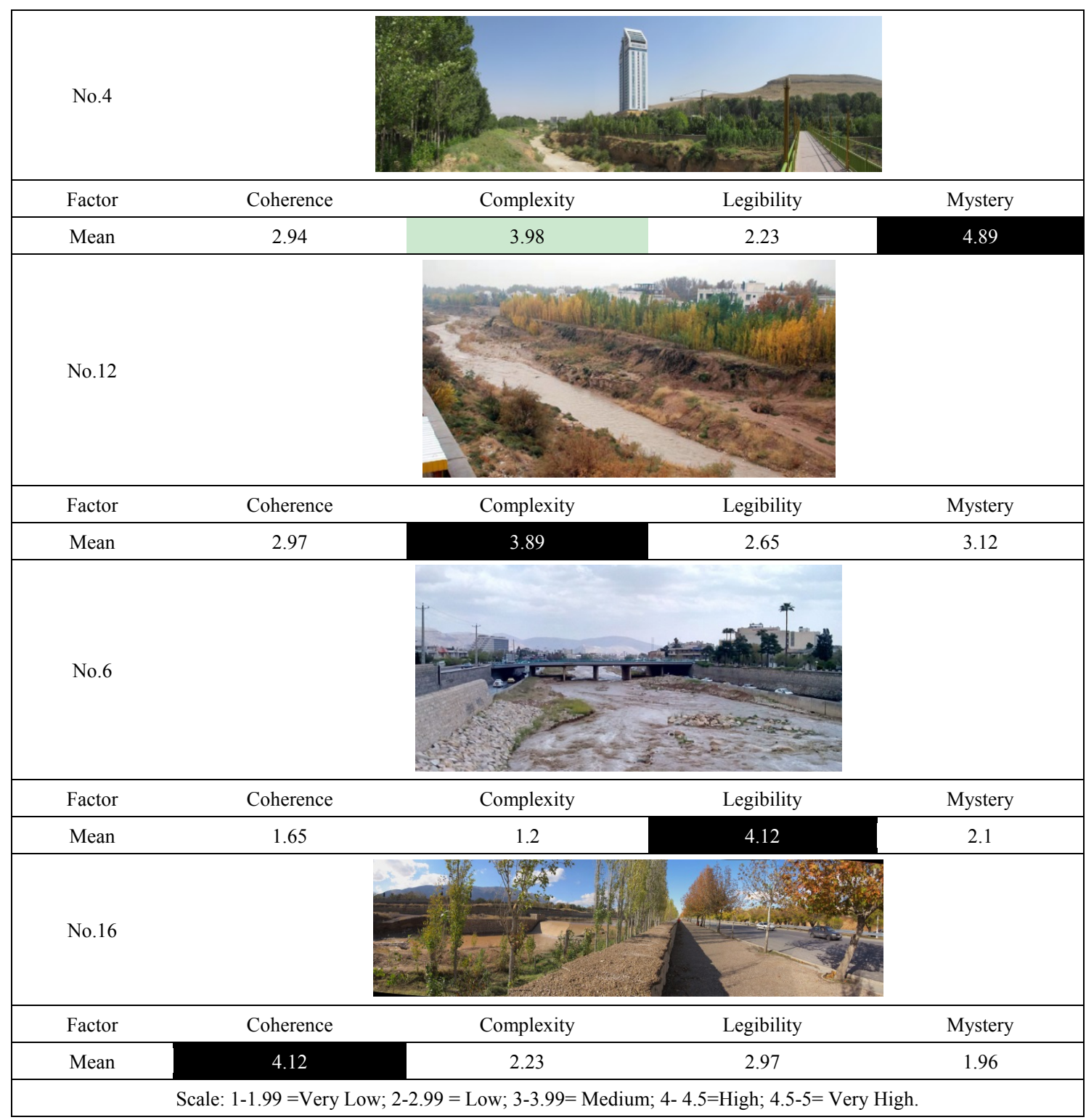

- Increasing the stream natural appearances (by planting trees, organic edges and water presence and etc.) could help citizens to have more coherent, complex, legible and mysterious preference of the stream scape;

- Most effects seem to be on immediate parameters which do not needs high level of accessibility, while its correlation to inferred parameters needs more accessible river front which lead to more experience and exploration in landscape;

- Gender, education, income and age do not have significant effects on public preferences matrix of this study;

- The study suggests that, naturalness can be added to Kaplan's' preference model in urban stream preference assessment, because of the great effects of populated conditions in these kinds of urban areas;

Further studies should be done in order to investigate the roll of vegetation planting order and its shape and size in preference of naturalness and other preferences attributes.

\section{Acknowledgements}

I would like to thank everyone who helps me during in field survey specially, Shiraz citizens. And also thanks the reviewers' useful comments.

\section{REFERENCES}

[1] Walsh, C.J., et al., The urban stream syndrome: current knowledge and the search for a cure. J. N. Am. Benthol. Soc., 2005. 24(3): p. 706-723. 
[2] Silva, J.B., F. Serdoura, and P. Pinto, Urban Rivers as Factors of Urban (Dis)integration, in 42nd ISoCaRP Congress. 2006. p. 1-14.

[3] Che, Y., et al., Assessing a riverfront rehabilitation project using the comprehensive index of public accessibility. Ecological Engineering, 2012. 40(1): p. 80-87.

[4] Le Lay, Y.-F., H. Piégay, and A. Rivière-Honegger, Perception of braided river landscapes: Implications for public participation and sustainable management. Journal of Environmental Management, 2013. 119(0): p. 1-12.

[5] $\mathrm{Wu}$, J. and Y. Xiao, River Corridor Greenway in Urban Environment: Perception and Use of East Ho Chung River Greenway in Guangzhou. Energy Procedia, 2011. 11: p. 2516 $-2523$.

[6] Nagpal, S. and A. Sinha, The Gomti Riverfront in Lucknow, India: Revitalization of a Cultural Heritage Landscape. Journal of Urban Design, 2009. 14(4): p. 489-506.

[7] Tapsell, S.M., Rivers and river restoration: a child's-eye view. Landscape Research, 1997. 22(2): p. 45-65.

[8] Prior, J., Urban river design and aesthetics: a river restoration case study from the UK. Journal of Urban Design, 2016. 21(4): p. $512-529$.

[9] Pinto, U. and B.L. Maheshwari, Community perspectives on managing health of peri-urban river system: evidence from the Hawkesbury-Nepean river catchment, Australia. Journal of Environmental Planning and Management, 2016. 59(7): p. $1257-1276$

[10] Podolak, K. and G.M. Kondolf, The Line of Beauty in River Designs: Hogarth's Aesthetic Theory on Capability Brown's Eighteenth-Century River Design and Twentieth-Century River Restoration Design. Landscape Research, 2016. 41(1): p. 149-167.

[11] Crow, T., T. Brown, and R. De Young, The Riverside and Berwyn experience: Contrasts in landscape structure, perceptions of the urban landscape, and their effects on people. Landscape and Urban Planning, 2006. 75(3-4): p. 282-299.

[12] Kearney, A.R., et al., Public perception as support for scenic quality regulation in a nationally treasured landscape. Landscape and Urban Planning, 2008. 87(2): p. 117-128.

[13] Book Reviews. Journal of the American Planning Association, 1986. 52(2): p. 224-243.

[14] Pourjafar, M., A. Sadeghi, and F. Ahmadi, Extension of Sustainable Designe Principles for the Natural Restoration of Shiraz Dry Sream. Environmental Sciences, 2010. 7(4): p. 193-202.

[15] Kaplan, R. and S. Kaplan, The Experience of Nature: A Psyvological Perspective. 1989: Combridge University Press.

[16] Oliver, K., Psychology in practice: environment, ed. H. Coolican. 2007: Hodder Arnold.

[17] van der Jagt, A.P.N., et al., Unearthing the picturesque: The validity of the preference matrix as a measure of landscape aesthetics. Landscape and Urban Planning, 2014. 124: p. 1-13.

[18] Tveit, M., A. Ode, and G. Fry, Key Concepts in A Framework for Analysing Visual Landscape Character. Lanscape Research, 2006. 31(3): p. 229-255.

[19] Lynch, K., The Image of The City. 1960: Publication of The Joint Center for Urban Studies.

[20] Arthur E and I. Stamps, Mystery, complexity, legibility and coherence: A meta-analysis. Journal of Environmental Psychology, 2004. 24(1): p. 1-16.

[21] Ikemi, M., The effects of mystery on preference for residential façades. Journal of Environmental Psychology, 2005. 25(2): p. $167-173$

[22] Mansouri, S.A. and A. Habibi, An Analysis of Factors Contributing to the Formation of Landscapes Ensuring Sustainable Environments A Case Study of the River Khoshk in Shiraz. Bagh-e-nazar, 2011. 7(15): p. 63-78. 